\title{
Kollektivität - Öffentliches Recht zwischen Gruppeninteressen und Gemeinwohl
}

\author{
Nora Markard"
}

Die diesjährige 52. Assistententagung widmete sich dem Thema „Kollektivität“ und fragte nach dem Öffentlichen Recht „zwischen Gruppeninteressen und Gemeinwohl“. Ausgerichtet wurde die Tagung von gleich drei Institutionen: der Juristischen Fakultät der Universität Hamburg, der Bucerius Law School und der HelmutSchmidt-Universität - Universität der Bundeswehr Hamburg. Drei Tage, drei Hochschulen, so kam jede dazu, sich mit ihren Örtlichkeiten bei herrlichem Frühlingswetter zu präsentieren.

Der Call for Papers hatte im Vorfeld der Tagung bereits die gesamte Breite des Themas entfaltet. Viele Aspekte fanden sich im Programm wieder, etwa Fragen des Ausgleichs kollektiver Interessen und der Solidarität durch Recht, plebiszitäre Partizipationsrechte und Repräsentationsansprüche, aber auch Themen wie Gemeinwohl, Generationengerechtigkeit und Nachhaltigkeit. Insbesondere die verfahrensmäßige Einbeziehung kollektiver Akteure wurde breit diskutiert. Wenig thematisiert wurden dagegen Fragen der Legitimation in heterogen verfassten Gemeinschaften, der Schutz repräsentationsschwacher Interessen und die Integration gesellschaftlicher Gruppen, während Vorträge mit theoriegeleitetem Zugriff eher rar gesät waren und eine explizit konzeptionelle Auseinandersetzung mit dem Begriff der Kollektivität fehlte.

Der Auftakt der eigentlichen Tagung, mit dem traditionellen Gongschlag zur Vertreibung der Habilitierten eingeleitet, wurde allgemein als fulminant wahrgenommen. Benjamin Rusteberg und Clemens Kaupa widmeten sich Kollektivinteressen in der Abwägung mit Individualrechten oder -interessen. Rusteberg beklagte die „strukturelle Disbalance“ zugunsten des Gemeinwohls in der Abwägung mit individuellen Freiheitsrechten und schlug stattdessen eine regelutilitaristische Betrachtung durch präzise Schutzbereichbestimmungen der speziellen Grundrechte vor, deren Einengung gegebenenfalls durch die Allgemeine Handlungsfreiheit aufgefangen werde. Rusteberg wandte sich damit gegen ein Optimierungsmodell wie bei Alexy, das individuelle Freiheiten stets unter einen Gemeinwohlvorbehalt stelle, und trat stattdessen für ein Dworkin'sches antagonistisches Trumpfmodell ein. Kaupa untersuchte die Gemeinwohlformulierung auf der Unionsebene durch den EuGH. Er zeigte am Beispiel der Viking-Entscheidung auf, dass der EuGH sich bei der Frage nach der Förderung oder Behinderung des Binnenmarkts als kollektivem Interesse stets für eine unter vielen konkurrierenden politischen Meta-Theorien entscheiden müsse, die je nach ökonomischem Modell zu unterschiedlichen rechtlichen Bewertungen führ-

* Dr. Nora Markard ist wissenschaftliche Mitarbeiterin am Sonderforschungsbereich 597 „Staatlichkeit im Wandel" an der Universität Bremen. 
ten. Dass es sich hier um eine Entscheidung handle, würde freilich regelmäßig nicht offengelegt. Das überwiegend herangezogene neoklassische Wirtschaftsmodell werde als das einzig denkbare präsentiert, obgleich mit der historischen Auslegung wohl viel eher ein keynesianisches Wirtschaftsverständnis zu vereinbaren wäre. Kaupa kritisierte die eigenmächtige Priorisierung eines bestimmten Wirtschaftsmodells durch den EuGH als Übertragung einer politischen Entscheidung an die juristischen Eliten, die jedoch nicht mit einer Absage an die europäische Integration zu beantworten sei; vielmehr entwickle der EuGH durchaus auch kooperative Strategien des Gesprächs mit den nationalen Parlamenten, z.B. im Gesundheitssektor. Dies sei der Weg, der künftig deutlich in den Mittelpunkt gerückt werden müsse.

Michaela Hailbronner leitete die Auseinandersetzung mit der Frage nach den Akteuren mit einem rechtsvergleichenden Vortrag ein. „Wer hat Angst vorm Kollektiv? " fragte sie und stellte die Einbeziehung kollektiver Akteure in der Rechtsdurchsetzung (public interest litigation in Indien), in der Verwaltung (USA) und der Rechtssetzung (Südafrika) vor. Sie kontextualisierte diese Mechanismen im Rahmen der je spezifischen Bedürfnisse, die sich in Deutschland freilich z.T. anders darstellten. Dennoch plädierte Hailbronner für eine deutsche Öffnung insbesondere im Bereich der Rechtssetzung und Verwaltung zur Erhöhung der demokratischen Legitimität, zumal in Deutschland keine agency capture durch übermächtige Lobby-Verbände zu befürchten sei. Hier seien auch aus Stuttgart 21 Konsequenzen zu ziehen, wo die Gelegenheit zur Einwendung - in fünfstelliger Zahl genutzt - und auch eine Verbandsklage nach NatSchG die Legitimationswirkung nicht erhöhen konnten. Hailbronner gestand zu, dass Verbände nicht an sich für die Allgemeinheit sprächen und zudem spezifische Interessen verträten; die Spezialisierung hätten sie aber den (demokratisch stärker legitimierten) Parteien voraus, deren inhaltliche Breite manchen Engagierten zu weit reiche. Angelika Günzel ergänzte diese Betrachtung durch eine detaillierte dogmatische Vorstellung der neuen Mechanismen zur Bürgerbeteiligung in Art. 11 EUV, der neben der „Zivilgesellschaft“ auch „repräsentative Verbände“ explizit erwähnt. Günzel bewertete diese Mechanismen kritisch, da die Repräsentativität der beteiligten Verbände bisher zweifelhaft sei und keine ausreichende Transparenz gewährleistet sei. Julia Schaarschmidt stellte die Möglichkeiten zur Einforderung von Gruppenrechten vor dem Afrikanischen Menschenrechtsgerichtshof vor, wo „peoples’ rights“ sogar im Wege der Popularklage verfolgt werden können.

Die von Hailbronner und Günzel bereits angesprochenen Verfahrensfragen setzten sich im dritten Block fort. Bilun Müller stellte die Bedeutung der EU-Mechanismen zur Öffentlichkeitsbeteiligung für das deutsche Verwaltungsrecht dar, für das im Lichte der Trianel-Entscheidung und der Aarhus-Konvention umfassender Änderungsbedarf bestehe. Thorsten Ricke widmete sich dem Netzausbaubeschleunigungsgesetz, das den Ausbau der Stromnetze im Hinblick auf erneuerbare Energien regelt 
und dabei umfassende Beteiligungsmöglichkeiten für kollektive Akteure vorsieht, die allerdings auf der Rechtsschutzebene nur eingeschränkt eingelöst werden.

Um Kollektivität als Motor dogmatischer Innovation ging es in den Vorträgen von Eva-Maria Schnelle und Dana Burchardt. Schnelle entwickelte Ansätze, um Elinor Ostroms polyzentrales Governance-Modell für die Allmende (aus dem Völkerrecht bekannt als das common heritage of mankind) rechtlich umsetzbar zu machen. Potential hierfür sah sie allerdings nur in kleinen, überschaubaren Kollektivitäten wie Gemeinden. Burchardt fragte rechtsphilosophisch und positivrechtlich nach der Rechtsträgerschaft zukünftiger Generationen und fand konkrete Anhaltspunkte in den völkerrechtlichen Prinzipien der Nachhaltigkeit und der intergenerationellen Gerechtigkeit.

Eher stiefmütterlich behandelte der fünfte Block das Solidaritätsprinzip. Peter Haversath sah mit Bezug auf den nationalen und supranationalen Bereich Solidarpflichten nur bei unverschuldeten Solidarfällen für gegeben an, was etwa im Fall Griechenland ausscheide. Auf der Basis seines Assekuranzmodell des Staates verneinte er ein ein übergreifendes Solidaritätsprinzip nicht nur für das GG, sondern auch zwischen den Staaten der EU, die lediglich zwischen den Unionsbürgern mittelten. Eine Solidargemeinschaft der Unionsbürger sei jedoch künstlich, es handle sich nicht um eine Erziehung zum Unionsbürger, sondern um eine rein rechtliche Integration. Boas Kümper schließlich stellte fest, dass das Recht der öffentlichen Ersatzleistungen mit dem Solidarprinzip wenig zu tun habe und auch nicht zu tun haben solle.

Um die Machtfrage innerhalb von Kollektiven ging es schließlich im sechsten und letzten Block - Tyrannei der Mehrheit, Macht der Minderheit - in erfrischend konkreter Form. Rafael Häcki führte in die schweizerischen Regelungen zur Verfassungsänderung durch Volksinitiativen ein, deren einzige materielle Schranke die Verletzung zwingenden Völkerrechts sei. Seit 2004 sei hier ein Paradigmenwechsel zu beobachten, indem zunehmend die Beschränkung der Rechte einzelner gesellschaftlicher Gruppen verfolgt würde. Die Ausschaffungs- und die Minarettinitiative stellten die rechtsanwendenden Behörden nun vor die Herausforderung, entweder die demokratisch geänderte Bundesverfassung anzuwenden oder sich an die menschenrechtlichen Vorgaben der EMRK zu halten. Häcki argumentierte, den Grundrechten und dem Rechtsstaatsprinzip müsse in solchen Konfliktfällen stets der Vorrang eingeräumt werden. Die deutsche Seite reagierte gewohnt unbescheiden und legte Häcki die Einführung einer Ewigkeitsklausel nach deutschem Modell ans Herz.

Roman Lehner diskutierte im Anschluss die deutschen direktdemokratischen Mechanismen. Während die repräsentative Demokratie Periodizität und einen institutionellen Ort brauche, sei die plebiszitäre Demokratie permanent und ortlos. Da der Volkswille in Plebisziten nicht einfach abgefragt, sondern erst gebildet werde, sei 
entscheidend, wer demokratisch legitimiert sei, die Frage zu stellen. Kampagnenfähigkeit erfordere finanzielle und soziale Ressourcen. Zudem enthielten sich viele aus Ahnungslosigkeit in Form einer „Selbstselektion der Inkompetentesten“, und zwar ebenfalls in schichtspezifischer Weise. Hierdurch entstehe eine Repräsentations-Partizipations-Lücke. Ein pluralismussicherndes Demokratiekonzept dürfe solche sozialen Selektionsmechanismen nicht verstärken. Lehner diskutierte verschiedene Lösungsmöglichkeiten, von einer Abstimmungspflicht bis hin zur digitalen Agora. Dieser erfreuliche Empiriebezug wurde allerdings nicht durchgehend begrüßt, vereinzelt wurde vielmehr eingewandt, das Recht könne sich nicht „an empirischen Befunden entlanghangeln“.

Für den Vorabend und die Festveranstaltung konnte das Tagungsteam illustre Gäste gewinnen. Wolfgang Hoffmann-Riem suchte nach Kollektiven und Kollektivitäten im World Wide Web und prüfte deren Regeln (z.B. netiquette) auf ihren rechtlichen Gehalt. Bundesjustizministerin Sabine Leutheusser-Schnarrenberger brach eine Lanze für den liberalen Individualismus im deutschen Recht und zeigte die Gefahren und Probleme der Einführung einer Verbandsklage auf. Insbesondere sei zweifelhaft, ob Verbände immer auch die Interessen der Allgemeinheit durchsetzten, wie sich am Beispiel der Waldschlösschenbrücke zeige. Zudem warnte sie vor Asymmetrien zwischen unterschiedlich durchsetzungsfähigen Gruppen, was einen gerechten Interessenausgleich verfehlen könne. EGMR-Richterin Angelika Nußberger beschrieb die Suche des EGMR nach einem Wertekonsens bei der Neuinterpretation von Konventionsrechten als identitätsstiftendes Kollektivitätsmoment im Europarat. „Wie definiert Europa die Werte, über die es sich definiert?", fragte Nußberger. Seit Marckx v. Belgium ${ }^{1}$ habe der Gerichtshof die margin of appreciation jeweils enger oder weiter gefasst, je nachdem ob sich der gesellschaftliche Wandel in der überwiegenden Zahl der Staaten auch rechtlich als „lebendiger Konsens“ niedergeschlagen habe. In $A, B$ and $C v$. Ireland ${ }^{2}$ habe der EGMR nun jedoch trotz eines wachsenden Konsenses in Richtung auf ein Abtreibungsrecht das irische Verbot für mit der Konvention vereinbar gefunden. Nußberger fragte, ob jede Rechtsentwicklung in den Konventionsstaaten auch immer zu einer entsprechenden Anhebung des Konventionsstandards führen müsse; der EGMR dürfe europäische Standards nicht von oben verordnen, sondern müsse Raum für individuelle Lösungen in vielgestaltigen europäischen Staaten behalten.

Insgesamt deckte die Tagung ein großes Spektrum an Fragen zum Thema ab und stellte auch immer wieder selbstverständlich Bezüge zum Völker- und Europarecht her, ohne dass es allerdings zu einer vertieften Auseinandersetzung mit dem Begriff der Kollektivität selbst kam. So wurden die Begriffe Kollektiv oder Kollektivität sehr 
unterschiedlich benutzt und selten definiert; für einige gehörte bereits Facebook dazu, andere sprachen eher über durch Ein- und Austritt regulierte Gemeinschaften. Eine konzeptionelle, übergreifend angelegte Behandlung des Themas steht damit noch aus.

Die Tagung wurde von der Verfasserin insgesamt als ausgesprochen gelungen empfunden, wozu natürlich auch die hervorragende Organisation, die wunderbare abendliche Hafenrundfahrt sowie spannende Führungen und Ortstermine das ihre beitrugen. Bemerkenswert war auch die entspannte und freundliche Atmosphäre der Konferenz. So wurde weit überwiegend zwar engagiert, aber nicht aggressiv diskutiert. Entsprechend knapp fiel auch die Aussprache am letzten Tag aus: minutenlange stehende Ovationen für das Tagungsteam. Dabei zeigte der vorher ausgegebene Evaluationsbogen, dass von Seiten des Organisationsteams durchaus Diskussionsbedarf bestanden hätte. Zum einen betraf dies die Kriterien bei der Auswahl der Papers, insbesondere die Berücksichtigung einer Diversität im Hinblick auf Qualifizierungsstufe, Geschlecht, Regionalität u.a. Zum anderen wurde nach dem Stellenwert des Rahmenprogramms gefragt, dessen deutlich gehobener Standard auch in den Pausengesprächen immer wieder Thema gewesen war. Aufgrund der sehr moderaten Teilnahmegebühr sind auch hierfür umfangreiche Gelder einzuwerben. Die Teilnahmezahl war daher wie schon im Vorjahr auf 250 begrenzt, über hundert weitere Personen hätten gern teilgenommen. Einige wenige hatten die Anreise auf eigenes Risiko auch ohne Anmeldung gewagt, was im Tagungsteam angesichts der strikten Begrenzung für großen Ärger sorgte.

Diese Fragen wird sich nun das nächste Tagungsteam stellen müssen. Mit einiger Spannung war die Verkündung des nächsten Tagungsortes erwartet worden, und unter großem Beifall und den Klängen eines mitgebrachten Alphorns übernahmen die Berner und Bernerinnen den berühmten Gong, mit dem schließlich die Hamburger Tagung endete. Bern lockt nun zur 53. Assistententagung in der ersten Februarwoche 2013 nicht nur mit Bergpanorama, Lebensqualität und entspanntem Humor, sondern auch mit einem dankbaren und theoriefreudigen Tagungsthema: „Das letzte Wort" fragt nach dem Verhältnis zwischen Legislative und Judikative. 